Chordomas are rare and low-grade malignant solid tumours, despite their histologically benign appearance, that arise in the bone from embryonic notochordal vestiges of the axial skeleton, a mesoderm-derived structure that is involved in the process of neurulation and embryonic development. Chordomas occurring in the skull base tend to arise in the basiocciput along the clivus. Three major morphological variants have been described (classical, chondroid, and atypical/dedifferentiated). The pathogenesis and molecular mechanisms involved in chordoma development remain uncertain. From a pathological standpoint, the microenvironment of a chordoma is heterogeneous, showing a dual epithelial-mesenchymal differentiation. These tumours are characterised by slow modality of biologic growth, local recurrence, low incidence of metastasis rates, and cancer stem cell (CSC) phenotype. The main molecular findings are connected with brachyury immunoexpression and activation of the downstream Akt and mTOR signalling pathways. The differentiation between typical and atypical chordomas is relevant because the tumoural microenvironment and prognosis are partially different. This review provides an insight into the recent and relevant concepts and histochemical markers expressed in chordomas, with special emphasis on dedifferentiated chordomas and their prognostic implications.

Key words: dedifferentiated, chordoma, skull base, immunophenotypic, tumor.

Contemp Oncol (Pozn) 2017; 21 (4): 267-273 DOI: https://doi.org/10.5114/wo.2017.72385

\section{Immunophenotypic features of dedifferentiated skull base chordoma: An insight into the intratumoural heterogeneity}

\author{
Kelvin Manuel Piña Batista ${ }^{1}$, Kenia Yoelvi Alvarez Reyes ${ }^{2}$, \\ Fátima Pérez Lopez ${ }^{3}$, Andrés Coca Pelaz ${ }^{4}$, Ivan Fernandez Vega ${ }^{5}$, \\ José Luis Llorente Pendás ${ }^{4}$, Antonio Saiz Ayala ${ }^{6}$, Aurora Astudillo ${ }^{5}$, \\ Jorge Andrés Nuñez Rojas ${ }^{4}$, Patricia Barrio Fernandez ${ }^{1}$
}

\author{
${ }^{1}$ Department of Neurosurgery, Hospital Universitario Central de Asturias, Oviedo, Spain \\ 2Department of Family and Community Medicine, Hospital Vital Alvarez Buylla, Oviedo, \\ Spain \\ ${ }^{3}$ Department of Radiology, Hospital Osatek Vitoria, Oviedo, Spain \\ ${ }^{4}$ Department of Otolaryngology and Skull Base Surgery, Hospital Universitario Central \\ de Asturias, Oviedo, Spain \\ DDepartment of Neuropathology, Hospital Universitario Central de Asturias, Oviedo, \\ Spain \\ ${ }^{6}$ Department of Neuroradiology, Hospital Universitario Central de Asturias, Oviedo, \\ Spain
}

\section{Introduction}

Chordomas are rare, aggressive, low-grade malignant solid tumours that arise in the bone from embryonic notochordal vestiges of the axial skeleton, a mesoderm-derived structure that is involved in the process of neurulation and embryonic development [1-14]. The sacrum represents the most common anatomical site of origin, accounting for about $50 \%$ of all cases, followed by the skull base region (spheno-occipital/nasal) in $35 \%[5,9,15-20]$. Skull base chordomas account for less than $0.2 \%$ of all intracranial neoplasms [5, 10, 21]. Recent studies suggest an incidence of chordoma of 0.08 cases per 100,000 with a peak incidence between 5060 years of age, which is higher in men over women $1: 0.6$ [3, 4, 15, 22-24].

Chordomas occurring in the skull base tend to arise in the basiocciput along the clivus, slightly inferior to the spheno-occipital synchondrosis or from the basisphenoid of the upper clivus, and typically are centred in the midline [6, 25-29]. Lateral growth of these neoplasms from their midline origin frequently results in secondary invasion of the cavernous sinus, which can be demonstrated radiologically in 54 to $75 \%$ of cases. Although these tumours are traditionally considered to be slow growing and histologically benign, they can be life-threatening. The disease is associated with poor survival due to the tendency to infiltrate the bone and high recurrence, carrying a dismal median survival time of approximately 6 years $[4,30]$. In a recent systematic meta-analysis with 467 patients, divided into three subgroups, taking into account their therapeutic management, overall survival, and disease-free survival: the overall survival (OS) and disease-free survival (DFS) rates after 5 years for patients with total resection but without radiotherapy were $92 \%$ and $94 \%$, respectively, and for those receiving adjuvant radiotherapy, the 5-year OS and DFS rates were $100 \%$ and $89 \%$, respectively. Among the patients undergoing partial resection without adjuvant radiotherapy, the 5-year OS and DFS rates were $41 \%$ and $45 \%$, respectively. However, in those receiving adju- 

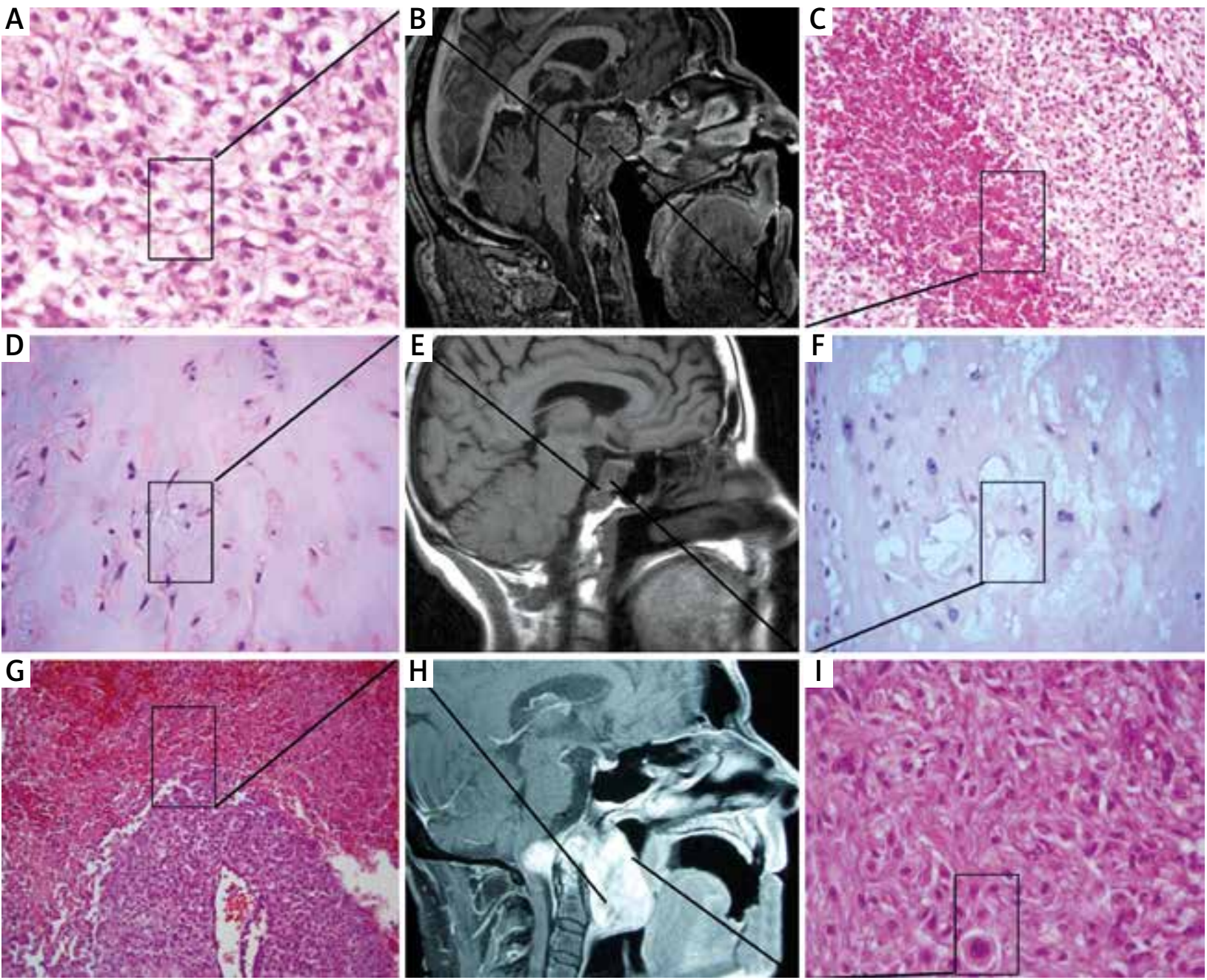

Fig. 1. Heterogeneity in chordoma variants: magnetic resonance images (MRI) and histopathology features, HE staining. A-C) Classic chordoma in a 76-year-old man. D-F) Chondroid chordoma in a 39-year-old man. B, E) Sagittal T1 MRI shows classic and chondroid chordomas, respectively. G-I) Dedifferentiated/atypical chordoma in a 62-year-old man. Images demonstrated intratumoural architectural and cytological heterogeneity in atypical chordoma. Different tumour areas of the same tumour showed variable characteristics. G) Necrotic area. H) preoperative sagittal T1-weighted MRI shows a huge dedifferentiated chordoma invading clivus, upper cervical spine, nasopharynx, and oropharynx. I) Atypical cells

vant radiotherapy after partial resection, the 5-year OS was $84 \%$ and DFS was $85 \%$ [31].

From a microscopic standpoint, chordoma is a moderately mucin-secreting cellular neoplasm, lobulated and semi-transparent, composed of vacuolated phys aliferous cells arranged singly and in cords within a myxoid stroma, as a distinguishing feature, completely surrounded by normal-appearing bone. Grossly, it can have a gelatinous surface, and inside the tumour is often soft with occasional translucent grey and blue areas, calcifications, focal haemorrhages, and cyst formations [4, 32, 33].

Regarding histopathology, three variants of chordoma are recognised (Fig. 1): conventional (classical or typical), chondroid, and dedifferentiated (atypical) [4, 6, 24, 29, 34-40]. Classical chordomas appear as soft, greyish, lobulated tumours composed of groups of cells separated by fibrous septa. They have round nuclei and an abundant, vacuolated eosinophilic and periodic acid
Schiff positive stain cytoplasm, embedded in hyaline cartilage-like stroma. Chondroid chordomas histologically display features of chordoma and chondrosarcoma, a malignant cartilage-forming tumour, and tend to occur almost exclusively in the skull base. Islands of cells characterise the chordoid areas with abundant eosinophilic cytoplasm arranged in cords, nest, and sheets with mucinous matrix. Physaliferous cells are occasionally observed. The chondroid areas consist of single cells set in an acellular hyaline-like extracellular matrix, usually surrounded by lacunar spaces. Chondroid chordoma shows a low-grade growth pattern, has a favourable long-term outcome, and tends to be less aggressive than typical chordomas. By contrast, dedifferentiated chordoma (variety accounts for approximately $5 \%$ of cases) exhibits high-grade behaviour and an aggressive clinical course, growing rapidly and being more likely to metastasize [26, 41-43]. While mitotic activity is rare in typical chordoma, poorly differentiated chordoma shows high mitotic 
activity (1-3/10 hpf - high power field) and high nuclear-to-cytoplasmic ratio with prominent nucleoli [34, 44]. The poorly differentiated chordoma is a particularly aggressive tumour with a predilection for the paediatric population and preferentially found in the skull base. Some authors postulated that dedifferentiated chordomas might arise secondary to sarcomatous transformation after radiotherapy $[3,22,45]$. The microenvironment of a chordoma is heterogeneous, showing a dual epithelial-mesenchymal differentiation. These tumours are characterised by slow modality of biologic growth, local recurrence, low incidence of metastasis rates, and cancer stem cell (CSC) phenotype [2, 4, 7, 22, 45, 46].

At present, the treatment paradigm of chordoma is wide en bloc or gross total surgical resection plus adjuvant proton-beam radiation [47, 48]; however, within the skull base, surrounding critical neurovascular structures and challenges in surgical access render clinical management of these patients difficult [8, 12, 35, 49]. Magnetic resonance imaging (MRI) is used to visualise tumour invasion by the presence of atypical radiographic patterns, including lateral extension, vascular encasement, cavernous invasion [11, 12, 21, 50, 51], suprasellar and subtemporal extension, and sphenoid-ethmoid-maxillary sinus invasion.

Generally, longer survival rates have been associated with more extensive tumour resection. Despite the histologically benign appearance, standard treatment remain ineffective and challenging because they are resistant to radiation therapy and because maximal surgical resection is impossible in most cases [4, 10, 52, 53]. In terms of chemotherapy, no regimen has been found to be very effective. Nonetheless, substantial heterogeneity is observed in patient outcome, including clinical course, treatment response, and survival.

Currently, the most useful prognostic factors in skull base chordoma are neurological deficits, preoperative Karnofsky performance scale score, metastasis, tumoural necrosis and apoptosis, Ki67 and p53 labelling indices, tumour differentiation, and unresectable status $[2,11,22,45,43]$.

The differentiation between typical and atypical chordomas is relevant because the tumoural microenvironment and prognosis are partially different. According to recent studies, the prognosis of patients with dedifferentiated chordoma is poor, and the majority of patients will experience local recurrence, or metastases, and will die of local progression within 6-12 months after diagnosis [2, 17, 34, 37, 42, 49, 54, 55].

A number of markers in chordomas have been discovered recently in approaching chordoma variants with their potential future diagnostic and prognostic utility. Recent studies have indicated that different types of biomarkers are relevant, such as brachyury, distinguishing between typical and dedifferentiated chordomas. This report provides an insight into the recent concepts and histochemical markers expressed in chordomas, with special emphasis on dedifferentiated chordomas and their prognostic implications.

\section{Pathologic mechanisms and biological markers in chordomas: chordoma dedifferentiation}

To date, studies have not revealed the fundamental mechanisms in the initiation and progression of chordomas. Contemporary research in chordoma biology and pathogenesis is scarce. Hence, accurate radiologic diagnosis and better understanding of the pathology of skull base chordomas are of valuable prognostic significance $[4,55,56]$. Nonetheless, the exact correlation of molecular data with specific pathological features in chordoma subtypes is not well understood. Common genetic lesions in chordomas, but not pathognomonic, are loss of heterozygosity (LOH) of chromosomes 1p36, 9p, 10q, and 17p [56]. Although chordomas are usually sporadic, a few familiar cases have been described $[8,56,57]$.

Chromosome loss of 1 p36 was found to be the most frequent genetic abnormality, occurring in $75-85 \%$ of both sporadic and familiar chordomas $[8,57,58]$. Several groups have postulated that the notochord development transcription factor brachyury (uncertainly expressed in almost all chordomas and suggested as an oncogenic driver) could be a novel discriminating biomarker for chordoma subtypes. Along with brachyury, receptor tyrosine kinases, including PDGFR (platelet-derived growth factor receptor) $-\alpha$ and $\beta$, EGFR (epidermal growth factor receptor), and c-Met (also called hepatocyte growth factor receptor), are frequently expressed in chordomas, resulting in the activation of the downstream Akt (also known as Protein Kinase B) and mTOR (mammalian Target of Rapamycin) signalling pathways [49, 59-61].

Recent data implicates cathepsin $\mathrm{K}$ as a relevant mediator of proteolytic degradation in chordoma spread, and a potential therapeutic target to reduce tumour spread and recurrence. Furthermore, chordomas express mainly higher levels of upregulated genes for collagen II, aggrecan, fibromodulin, cartilage-linking protein, transcription factor SOX9, fibronectin, MMP9 (matrix metalloproteinase 9), MMP19, CD24 antigen, podoplanin, survivin, periplakin, and discoidin domain receptor 1 [30, 41, 52, 62-66]. Traditionally, CK (cytokeratin), EMA (epithelial membrane antigen), and S-100 protein are the immunohistochemical markers that have been used to substantiate a diagnosis of a chordoma [63].

At the chromosomal level, atypical chordomas are characterised by polyploidy complex karyotype that is associated with aggressive biological behaviour and a dismal prognosis [54]. Expression of traditional markers such as Ki67, a proliferative index that is expressed at all stages of mitosis except for G0, and p53 correlated with the recurrence of intracranial chordomas [10, 30].

Although chordomas are considered to be slow-growing notochordal cell tumours, dedifferentiated chordomas behave like sarcomas due to their anaplastic/sarcomatoid transformation, occurring in a multistep process that collectively involves molecular and oncogenic alterations [3, 4, 41, 52, 67, 68]. However, the molecular determinants contributing to the pathogenic evolution of a dedifferentiated chordoma and its relationship with classical chordomas are largely unknown. Accumulating 
evidence has indicated that the dedifferentiated chordoma can develop de novo from notochordal remnants or years later from precursor classical chordoma. A recent retrospective cohort study of 25 chordoma patients found that adverse patient outcome is significantly associated with spindle cell sarcomatoid feature and intratumoural necrosis [68].

Until now, no molecular features have been proven conclusive in establishing prognosis in skull base chordomas, but the protein expression levels of cadherins, catenins [69-72], matrix metalloproteinases (MMPs), cathepsin $\mathrm{K}$, cathepsin B, and urokinase-type plasminogen activator are related to the invasion of skull base chordoma, and these levels may affect treatment effect and consequently affect the prognosis. Cathepsin K, a well-known osteoclastogenesis marker, is a member of the papain family of cysteine proteases that is expressed by osteoclasts, which plays an important role in bone resorption, and is associated with tumour invasion and poor prognosis. Cathepsin K overexpression in chordomas is implicated in invasion and migration of the tumour $[53,59]$. Unfortunately, there are no data about the role of cathepsin $\mathrm{K}$ on dedifferentiated chordomas.

Jambhekar et al. in recent study found that dedifferentiated chordomas possess areas of conventional chordoma, with CK and vimentin immunostaining, and juxtaposed with unequivocal sarcomatous elements, most frequently resembling malignant fibrous histiocytoma, that revealed EMA positivity [63]. Furthermore, in an immunohistochemical and DNA flow cytometric research of four dedifferentiated chordomas, authors found that spindle cells had an aneuploidy-multiploid DNA content and showed weak staining for cytokeratin (CK) and EMA, but increased in the staining for vimentin, as compared with the conventional chordoma regions in the same tumours [73]. Conversely, Gil et al., through a cytogenetic analysis of three variants of clival chordoma, found that sarcomatous areas of dedifferentiated chordomas are negative for the epithelial markers such as CK and EMA [73]. A more recent study reported that conventional and transitional chordomas were immunoreactive for EMA, CK, and S-100, despite an absence of the same markers in the centre of the sarcomatous areas [42]. Choi et al. reported a case report of an atypical chordoma in which most of the epithelial components of classical chordoma were strongly positive for CK, EMA, and S-100 protein, although in the sarcomatous elements there was negative staining for CK and EMA, but immunoreactive for vimentin and alpha1-anti-chymo-trypsin [74].

Recent chordoma cell line Western blot and immunohistochemistry analysis revealed that dedifferentiated cells expressed high levels of platelet-derived growth factor, high surface expression of cancer stem cell marker CXCR4, but absent or low level of brachyury and CKs [52].

Brachyury is apparently the marker having the best sensitivity (98\%) and specificity (100\%), while the vast majority of markers have a variable immunoexpression
[62]. Brachyury, meaning short tail in Greek, is the transcription factor protein product of a T-box gene, which plays an important role in the formation of the normal mesoderm and notochord in humans [41, 62], and in epithelial tumours. Brachyury overexpression promotes epithelial-to-mesenchymal transition (EMT), a reversible biological process that occurs in epithelial cells, inducing stem-like features such as motility, invasiveness [70], and resistance to conventional genotoxic agents $[66,72]$. Paradoxically, silencing of brachyury expression in brachyury-positive human neoplasm cells induces downregulation of mesenchymal markers and upregulation of epithelial markers, with concomitant loss of cell migration and extracellular membrane (ECM) invasion [66]. Nevertheless, based on retrospective analyses of patients with colorectal, breast [75], lung, and hepatocellular carcinoma, brachyury expression has been associated with poor prognosis $[41,61,66,76]$.

Although studies have shown that brachyury overexpression is essential for the survival chordoma cells, and have allowed their differentiation from chondrosarcomas, with relatively high sensitivity and specificity, is not associated with prognosis [33]. Interestingly, chondrosarcomas are mesenchymal neoplasms with positive staining for vimentin and S-100 protein but frequently do not express CK or EMA, and have good prognosis when treated with surgery and proton beam irradiation [26]. Surprisingly, brachyury triggers overexpression of EMT-associated proteins including $\mathrm{N}$-cadherin, snail, slug, and twist families [61]. In lung cancer, brachyury induces upregulation of $\mathrm{N}$-cadherin and vimentin [65]. Twist, a protein with a basic helix-loop-helix structure and EMT regulator, acts independently of snail to suppress $\mathrm{E}$-cadherin and to upregulate $\mathrm{N}$-cadherin, and has been found to be elevated in various types of neoplasm, including gastric cancer and melanoma [66]. Snail is a primary suppressor of E-cadherin expression, and encodes a transcriptional repressor that, along with transcription factors of the ZEB, slug, and twist families, is a mediator of EMT [61]. Transforming growth factor $\beta$ (TGF- $\beta$ ) induces the nuclear localisation of snail through Smad2/3 pathways [77, 78]. Interestingly, chordoma cells express many of the markers of mesenchymal cells typically induced with brachyury upregulation in other tumour types, including decreased E-cadherin levels and increased $\mathrm{N}$-cadherin and vimentin levels [72]. In fact, a study of clival chordomas has shown that E-cadherin and $\mathrm{N}$-cadherin expression presented an inverse immunoexpression. Thereby, E-cadherin to $\mathrm{N}$-cadherin shift plays a pivotal role in cancer progression and dismal prognosis, probably caused by the transition from a less invasive to an aggressive tumour phenotype [70]. Strikingly, atypical chordomas demonstrate negative or low levels of EMA, CK, and brachyury expression [52]. Conversely, Barresi et al. in a previous study found that brachyury expression represents a unique specific diagnostic marker for local and metastatic atypical chordoma [79]. In summary, regarding recent and contradictory 
data about brachyury expression, we can conclude that chordomas are a heterogeneous pathology, and the mechanism by which brachyury overexpression promotes epithelial-to-mesenchymal transition involved in motility and invasiveness as a negative feature of neoplasm development is ambiguously absent or scantly expressed in atypical chordomas, and needs further investigations $[3,14,24,36,76,78,80]$.

Apart from the distinction between typical and atypical chordomas, an emerging issue is the definition of prognostic aspects based on molecular and histopathological features, which might help clinicians to define the best therapeutic strategies.

The authors declare no conflict of interest.

\section{References}

1. Soo MY. Chordoma: Review of clinicoradiological features and factors affecting survival. Australas Radiol 2010; 45: 427-34.

2. Ferraresi V, Nuzzo C, Zoccali C, et al. Chordoma: clinical characteristics, management and prognosis of a case series of 25 patients. BMC Cancer 2010; 10: 22.

3. Frankl J, Grotepas C, Stea B, Lemole GM, Chiu A, Khan R. Chordoma dedifferentiation after proton beam therapy: a case report and review of the literature. I Med Case Rep 2016; 10: 280.

4. Walcott BP, Nahed BV, Mohyeldin A, Coumans JV, Kahle KT, Ferreira MJ. Chordoma: current concepts, management, and future directions. Lancet Oncol 2012; 13:e69-e76.

5. Lanzino G, Dumont AS, Lopes MB, Laws ER Jr. Skull base chordomas: overview of disease, management options, and outcome. Neurosurg Focus 2001; 10: E12.

6. Erdem E, Angtuaco EC, Van Hemert R, Park JS, Al-Mefty O. Comprehensive review of intracranial chordoma. RadioGraphics 2003; 23: 995-1009.

7. Patel NP, Mhatre AN, Lalwani AK. Molecular pathogenesis of skull base tumors. Otol Neurotol 2004; 25: 636-43.

8. Sun X, Hornicek F, Schwab JH. Chordoma: an update on the pathophysiology and molecular mechanisms. Curr Rev Musculoskelet Med 2015; 8: 344-52.

9. Firooznia H, Pinto RS, Lin JP, Baruch HH, Zausner J. Chordoma: radiologic evaluation of 20 cases. AJR Am J Roentgenol 1976; 127: 797-805.

10. PalliniR,Maira G, PiercontiF,FalchettiML,Alvino E, Crimino-RealeG, Fernandez E, D`Ambrosio E, Larroca LM. Chordoma of the skull base: predictors of tumor recurrence. J Neurosurg 2003; 98: 812-22.

11. Sekhar LN, Pranatartiharan R, Chanda A, Wright DC. Chordomas and chondrosarcomas of the skull base: results and complications of surgical management. Neurosurg Focus 2001; 10: E2.

12. Colli BO, Al-Mefty O. Chordomas of the skull base: follow-up review and prognostic factors. Neurosurg Focus 2001; 10: E1.

13. Beschorner R, Koerbel A, Schittenhelm J, Kaminsky J, Loewenheim H, Bueltamann E, Tatagiba M, Meyermann R, Wehrmann M. 47-year-old woman with a clival mass. Brain Pathol 2008; 18: 100-2.

14. Miettinen M, Wang Z, Lasota J, Heery C, Schlom J, Palena C. Nuclear brachyury expression is consistent in chordoma, common in germ cell tumors and small cell carcinomas, and rare in other carcinomas and sarcomas: An immunohistochemical study of 5229 cases. Am J Surg Pathol 2015; 39: 1305-12.

15. Hashim H, Rosman AK, Abdul Aziz A, Rogiah AK, Bakar NS. Atypical clival chordoma in an adolescent without imaging evidence of bone involvement. Malays I Med Sci 2014; 21: 78-82.
16. Moriki T, Takahashi T, Wada M, Ueda S, Ichien M, Miyazaki E. Chondroid chordoma: fine needle aspiration cytology with histopathological, immunohistochemical, and ultrastructural study of two cases. Diagn Cytopathol 1999; 21: 335-9.

17. Choi D, Gleeson M. Surgery for Chordomas of the craniocervical junction: lessons learned. Skull Base 2010; 20: 41-5.

18. Uda T, Ohata K, Takami T, Hara M. An intradural skull base chordoma presenting with acute intratumoral hemorrhage. Neurol India 2006; 54: 306-7.

19. Chapman PR, Shah R, Curé JK, Bag AK. Petrous apex lesions: pictorial review. AJR Am J Roentgenol 2011; 196 (3 Suppl): WS26-37 Quiz S40-3. doi: 10.2214/AJR.10.7229.

20. Chugh R, Tawbi H, Lucas DR, Biermann JS, Schuetze SM, Baker LH. Chordoma: the nonsarcoma primary bone tumor. Oncologist 2007; 12: 1344-50.

21. Koutourousiou M, Snyderman CH, Fernandez-Miranda J, Gardner PA. Skull base chordomas. Otolaryngol Clin North Am 2011; 44: 1155-71.

22. Aydemir E, Bayrak OF, Sahin F, Atalay B, Kose GT, Ozen M, Sevli $S$, Dalan AB, Yalvac ME, Dogruluk T, Türe U. Characterization of cancer stem-like cells in chordoma. J Neurosurg 2012; 116: 81020.

23. Rutz HP, Weber DC, Sugahara S, et al. Extracranial chordoma: outcome in patients treated with function-preserving surgery followed by spot-scanning proton beam irradiation. Int I Radiat Oncol Biol Phys 2007; 67: 512-20.

24. Khurram SA, Biswas D, Fernando M. A parapharyngeal soft tissue chordoma presenting with synchronous cervical lymph node metastasis: an unusual Presentation. Head Neck Pathol 2016; 10: 400-4.

25. Gonzalez-Martinez JA, Guthikonda M, Vellutini E, Zamorano L, Li Q, Kupski W, Diaz FG. Intradural invasion of chordoma: two case reports. Skull Base 2002; 12: 155-61.

26. Richardson MS. Pathology of skull base tumors. Otolaryngol Clin North Am 2001; 34: 1025-42.

27. Falcioni M, Taibah A, Caruso A. Clival chordoma mimicking a trigeminal schwannoma. Otol Neurotol 2001; 22: 706-7.

28. Navas M, Martinez P, Shakur S, Barbosa A, Barcena E, Gordillo C, Fraga J, Blanco C, Sola RG. Intrasellar chordoma associated with a primitive persistent trigeminal artery. Turk Neurosurg 2015; 25: 146-53.

29. Clark WC, Robertson JH, Lara R. Chondroid chordoma. J Neurosurg 1982; 57: 842-5.

30. Sakai K, Hongo K, Tanaka Y, Nakayama J. Analysis of immunohistochemical expression of $\mathrm{p} 53$ and the proliferation marker Ki-67 antigen in skull base chordomas: relationships between their expression and prognosis. Brain Tumor Pathol 2007; 24: 57-62.

31. Amit M, Na'ara S, Binenbaum Y, Billan S, Sviri G, Cohen JT, Gil Z. Treatment and outcome of patients with skull base chordoma: a meta-analysis. J Neurol Surg B Skull Base 2014;75: 383-90.

32. Pamir MN, Ozduman K. Tumor-biology and current treatment of skull-base chordomas. Adv Tech Stand Neurosurg 2008; 33: 35-129.

33. Zhang L, Guo S, Schwab JH, et al. Tissue microarray immunohistochemical detection of brachyury is not a prognostic indicator in chordoma. PLoS ONE 2013; 8: e75851.

34. Pamir MN, Ozduman K. Analysis of radiological features relative to histopathology in 42 skull-base chordomas and chondrosarcomas. Eur J Radiol 2006; 58: 461-70.

35. Yasuda M, Bresson D, ChibbaroS, Cornelius JF, Polivka M, Feuvret L, Takayasu M, George B. Chordomas of the skull base and cervical spine: clinical outcomes associated with a multimodal surgical resection combined with proton-beam radiation in 40 patients. Neurosurg Rev 2012; 35: 171-83.

36. Kreshak J, Larousserie F, Picci P, Boriani S, Mirra J, Merlino B, Brunocilla E, Vanel D. Difficulty distinguishing benign notochordal cell tumor from chordoma further suggests a link between them. Cancer Imaging. 2014; 14: 4. doi: 10.1186/14707330-14-4. 
37. Bell D, Raza SM, Bell AH, Fuller GN, DeMonte F. Whole-transcriptome analysis of chordoma of the skull base. Virchows Arch 2016; 469: 439-49.

38. Tamaki M, Aoyagi M, Kuroiwa T, Yamamoto M, Kishimoto S, Ohno K. Clinical course and autopsy findings of a patient with clival chordoma who underwent multiple surgeries and radiation during a 10-year period. Skull Base2007; 17: 331-40.

39. Vrionis FD, Kienstra MA, Rivera M, Padhya TA. Malignant tumors of the anterior skull base. Cancer Control 2004; 11: 144-51.

40. Nishiguchi T, Mochizuki K, Ohsawa M, Inoue T, Kageyama K, Su zuki A, Takami T, Miki Y. Differentiating benign notochordal cell tumors from chordomas: radiographic features on mri, ct, and tomography. AJR Am J Roentgenol 2011; 196: 644-50.

41. Gulluoglu S, Turksoy O, Kuskucu A, Ture U, Bayrak OF. The molecular aspects of chordoma. Neurosurg Rev 2015; 39: 185-96.

42. Saito A, Hasegawa T, Shimoda T, Toda G, Hirohashi S, Tajima G, Moriya Y. Dedifferentiated chordoma: a case report. Jpn J Clin Oncol 1998; 28: 766-71.

43. Tauziède-Espariat A, Bresson D, Polivka M, et al. Prognostic and therapeutic markers in chordomas: a study of 287 tumors. J Neuropathol Exp Neurol 2016; 75: 111-20.

44. Naka T, Boltze C, Samii A, et al. Skull base and nonskull base chordomas. Cancer 2003; 98: 1934-41.

45. Heo J, Cho SJ. A case of giant skull base chondroma. Brain Tumor Res Treat 2014; 2: 92-4.

46. Park TS, Donnenberg VS, Donnenberg AD, Zambidis ET, Zimmerlin L. Dynamic interactions between cancer stem cells and their stromal partners. Curr Pathobiol Rep 2014; 2: 41-52.

47. Igaki H, Tokuuye K, Okumura T, et al. Clinical results of proton beam therapy for skull base chordoma. Int J Radiat Oncol Biol Phys 2004; 60: 1120-6.

48. Weber DC, Rutz HP, Pedroni ES, Bolsi A, Timmermann B, Verwey J, Lomax AJ, Goitein G. Results of spot-scanning proton radiation therapy for chordoma and chondrosarcoma of the skull base: The Paul Scherrer Institut experience. Int J Radiat Oncol Biol Phys 2005; 63: 401-409.

49. Erkmen CP, Barth RJ Jr, Raman V. Case report: Successful treatment of recurrent chordoma and bilateral pulmonary metasta ses following an 11-year disease-free period. Int I Surg Case Rep 2014; 5: 424-7.

50. Di Maio S, Yip S, Al Zhrani GA, Alotaibi FE, Al Turki A, Kong E, Rostomily RC. Novel targeted therapies in chordoma: an update. Ther Clin Risk Manag 2015; 11: 873-83.

51. Tamaki N, Nagashima T, Ehara K, Motooka Y, Barua KK. Surgical approaches and strategies for skull base chordomas. Neurosurg Focus 2001;10: E9.

52. Kim JY, Lee J, Koh JS, Park MJ, Chang UK. Establishment and characterization of a chordoma cell line from the tissue of a patient with dedifferentiated-type chordoma. J Neurosurg Spine 2016; 25: 626-35.

53. Tian K, Ma J, Wang L, et al. Expression of cathepsin k in skull base chordoma. World Neurosurg 2017; pii: S1878-8750(17)30172-9.

54. Gil Z, Fliss DM, Voskoboinik N, Leider-Trejo L, Spektor S, Yaron Y, Orr-Urtreger A. Cytogenetic analysis of three variants of clival chordoma. Cancer Genet Cytogenet 2004; 154: 124-30.

55. Wang HF, Ma HX, Ma CY, Luo YN, Ge PF. Sellar chordoma presenting as pseudo-macroprolactinoma with unilateral third cranial nerve palsy. Chin J Cancer Res 2012; 24: 167-70.

56. Longoni M, Orzan F, Stroppi M, Boari N, Mortini P, Riva P. Evaluation of 1 p36 markers and clinical outcome in a skull base chordoma study. Neuro Oncol 2008; 10: 52-60.

57. Wang K, Wu Z, Tian K, Wang L, Hao S, Zhang L, Zhang J. Familial chordoma: A case report and review of the literature. Oncol Lett 2015; 10: 2937-40.

58. Horbinski C, Oakley GJ, Cieply K, Mantha GS, Nikiforova MN, Dacic S, Seethala RR. The prognostic value of Ki-67, p53, epidermal growth factor receptor, 1p36, 9p21, 10q23, and 17p13 in skull base chordomas. Arch Pathol Lab Med 2014; 134: 1170-6.

59. Naka T, Boltze C, Samii A, et al. Expression of c-MET, low-molecular-weight cytokeratin, matrix metalloproteinases-1 and -2 in spinal chordoma. Histopathology 2009; 54: 607-13.
60. Lee DH, Zhang Y, Kassam AB, et al. Combined PDGFR and HDAC inhibition overcomes PTEN disruption in chordoma. Loeb DM, editor. PLOS ONE 2015; 10: e0134426.

61. Nibu Y, José-Edwards DS, Di Gregorio A. From notochord formation to hereditary chordoma: the many roles of brachyury. Biomed Res Int 2013; 2013: 826435.

62. Oakley GJ, Fuhrer K, Seethala RR. Brachyury, SOX-9, and podoplanin, new markers in the skull base chordoma vs chondrosarcoma differential: a tissue microarray-based comparative analysis. Mod Pathol 2008; 21: 1461-9.

63. Jambhekar NA, Rekhi B, Thorat K, Dikshit R, Agrawal M, Puri A Revisiting chordoma with brachyury, a "new age" marker: analysis of a validation study on 51 cases. Arch Pathol Lab Med 2010; 134: 1181-7.

64. Bayrakli F, Guney I, Kilic T, Ozek M, Pamir MN. New candidate chromosomal regions for chordoma development. Surgical Neurol 2007; 68: 425-30.

65. Hu Y, Mintz A, Shah SR, Quiñones-Hinojosa A, Hsu W. The FGFR/ MEK/ERK/brachyury pathway is critical for chordoma cell growth and survival. Carcinogenesis 2014; 35: 1491-9.

66. Fernando RI, Litzinger M, Trono P, Hamilton DH, Schlom J, Palena C. The T-box transcription factor Brachyury promotes epithelial-mesenchymal transition in human tumor cells. J Clin Invest 2010; 120: 533-44

67. Chang C, Chebib I, Torriani M, Bredella M. Osseous metastases of chordoma: imaging and clinical findings. Skeletal Radiol 2017; 46: 351-8

68. Yakkioui Y, Temel Y, Creytens D, Jahanshahi A, Fleischeuer R, Santegoeds RG, Van Overbeeke JJ. A comparison of cell-cycle markers in skull base and sacral chordomas. World Neurosurg 2014; 82: e311-318.

69. Wu Z, Wang L, Guo Z, Wang K, Zhang Y, Tian K, Zhang J, Sun W, Yu C. Experimental study on differences in clivus chordoma bone invasion: an itraq-based quantitative proteomic analysis. PLOS ONE 2015; 10: e011952.

70. Triana A, Sen C, Wolfe D, Hazan R. Cadherins and catenins in clival chordomas. Am J Surg Pathol 2005; 29: 1422-34.

71. Feng Y, Zhang Q, Wang Z, Yan B, Wei W, Li P. Overexpression of the BMP4/SMAD signaling pathway in skull base chordomas is associated with poor prognosis. Int J Clin Exp Pathol 2015; 8: 8268-75.

72. Heery CR. Chordoma: The quest for better treatment options. Oncol Ther 2016 ; 4: 35-51.

73. Hruban RH, Traganos F, Reuter VE, Huvos AG. Chordomas with malignant spindle cell components: a DNA flow cytometric and immnunohistochemical study with histogenetic implications. Am J Pathol 1990; 137: 435-47

74. Choi YJ, Kim TS. Malignant fibrous histiocytoma in chordoma-immunohistochemical evidence of transformation from chordoma to malignant fibrous histiocytoma. Yonsei Med 1994; 35: 239-43.

75. Shakir SI, Pelmus M, Florea A, Boileau JF, Guiot MC, Di Maio S, Muanza TM. Synchronous metastatic skull base chordoma to the breast: case report and literature review. Curr Oncol 2016; 23: 154-7.

76. Wang K, Tian K, Wang L, et al. Brachyury: A sensitive marker, but not a prognostic factor, for skull base chordomas. Mol Med Report 2015; 12: 4298-304

77. Cortini M, Massa A, Avnet S, Bonuccelli G, Baldini N. Tumor-activated mesenchymal stromal cells promote osteosarcoma stemness and migratory potential via il-6 secretion. PLoS One 2016; 11: e0166500-22.

78. Deniz ML, KI TR, Almaata D, Kurtkaya Z, Sav A, Pamir MN. Expression of growth factors and structural proteins in chordomas: basic fibroblast growth factor, transforming growth factor, and fibronectin are correlated with recurrence. Neurosurgery 2002; 51: 753-60.

79. Barresi V, Ieni A, Branca G, Tuccari G. Brachyury: A diagnostic marker for the differential diagnosis of chordoma and hemangioblastoma versus neoplastic histological mimickers. Dis Markers 2014; 2014: 514753. 
80. Lagman C, Varshneya K, Sarmiento JM, Turtz AR, Chitale RV. Proposed diagnostic criteria, classification schema, and review of literature of notochord-derived ecchordosis physaliphora. Cureus 2016; 8: e547.

\section{Address for correspondence}

Kelvin Manuel Piña Batista

Department of Neurosurgery

Hospital Universitario Central de Asturias

Oviedo, Spain

e-mail: pineappledr@gmail.com

Submitted: 14.05 .2017

Accepted: $\quad 26.08 .2017$ 